\title{
Surgical Resident Attrition and the Menninger Morale Curve
}

\author{
Jack Contessa $^{1}$, Tassos Kyriakides ${ }^{2}$ \\ ${ }^{1}$ Department of Surgery, Hospital of Saint Raphael, New Haven, USA \\ ${ }^{2}$ Department of Internal Medicine, Yale University School of Medicine, New Haven, USA \\ E-mail: jcontessa@srhs.org, tassos.kyriakides@yale.edu \\ Received June 23, 2011; revised July 20, 2011; accepted August 11, 2011
}

\begin{abstract}
BACKGROUND: Attrition in surgical residency programs continues to be a significant challenge. Approximately $20 \%$ of residents who begin a categorical surgery residency fail to complete it. A number of studies speculated reasons for this including work hours, life style, family pressures, and resident feelings of inadequacy including fear of termination. To date no research has been conducted investigating the relationship between resident morale and attrition. This study sought to determine if this linkage exists in surgery residents. METHODS: The Morale Assessment in General Practice Index (MAGPI) was administered to 21 PGY 1, 2, 3, and 5 surgical residents to assess level of morale. Non-parametric methods were carried out to assess if there were differences in morale among the four PGY groups. Additionally, analyses of the four factors comprising the MAGPI were also conducted. RESULTS: Although differences did not reach statistical significance, analysis of the data reveals that residents demonstrate different trends in their levels of morale based on the amount of time they spend in a residency and in a way that approximates the morale curve described by W. Walter Menninger, M.D. Additionally, two of the four factors comprising the MAGPI also indicate trends similar to that described by the Menninger morale curve. CONCLUSIONS: Although no statistically significant results were achieved, the data reveal trends that approximate shifts in morale similar to those described by the Menninger morale curve, with residents at the PGY 2 and 3 levels presenting lower morale levels than at the PGY 1 and 5 levels. This may be due in part to the size of the population studied. Future research should be continued in this area with a larger sample size.
\end{abstract}

Keywords: Morale Assessment in General Practice Index (MAGPI), Menninger Morale Curve, Surgery Resident Attrition

\section{Introduction}

The attrition rate for general surgery residents continues to be a significant challenge for programs. Studies done at the American Board of Surgery suggest that nationally 20 percent or more of residents who begin a categorical surgery residency fail to complete it [1]. Similar outcomes were reported in a single institution study of 795 medical graduates, 43 of which entered general surgery residency programs with 7 discontinuing their training (16\% attrition rate) [2]. In their study of why residents leave general surgery, Dodson and Webb reported that although research in this area is limited, there have been a number of studies that documented a fairly consistent attrition rate between $14 \%$ to $23 \%$ [3]. In a research study investigating attrition rates in residents entering US obstetrics and gynecology residencies, McAlister et al. quoted an ACGME census data report for the 2004 2005 academic year which identified general surgery attrition rate at 5.8\%-higher than obstetrics and gynecology (5.1\%), family medicine (4.7\%), and internal medicine (2.1\%) [4]. Additionally, Longo et al. reported an overall annual attrition rate of $6.7 \%$ in their residency program over a 20 year period [5].

Surgery continues to have one of the highest attrition rates of all graduate medical education programs, which is of particular concern since it is predicted that there will be a substantial shortage of general surgeons. Data reported through 2005 show the population of general surgeons across the country has already decreased from 
7.68 per 100,000 population in 1981 to 5.69 per 100,000 population in 2005-a decline of almost 26\% [6]. Furthermore, it appears implementation of work hour restrictions has paradoxically exacerbated the attrition problem. One national study of general surgery residency programs reported that the percentage of residents lost through attrition increased from $29 \%$ to $44 \%$ since institution of the 80-hour workweek [7].

A number of studies have speculated reasons for resident attrition including work hours, lifestyle, pressures from family, resident feelings of inadequacy along with the belief that they will be terminated, economic considerations, and possible disconnect by medical students about the reality of a general surgery work experience. Additionally, it appears that the highest attrition rate occurs during the first two years of training with $70 \%$ or more taking place during this timeframe [5,7]. This is confirmed in at least two other studies; one reported residents who left their program voluntarily often left during or after the PGY 1 or PGY 2 year [3] and the second study which indicated that $75 \%$ of all attrition occurred at these levels [8]. In her survey of nearly 4500 general surgery residents regarding attitudes, training experiences, and professional expectations, Yeo reported overall satisfaction varied significantly across training years with the lowest reported level in the PGY 2 year (82.8\%) and the highest level in the PGY 5 year (89.7\%). Also, reports of having considered leaving training differed significantly with the highest in the PGY 2 year (19.2\%) and the lowest in the PGY 5 year (7.2\%). Furthermore, PGY 2 residents reported the lowest level of feeling that they could turn to faculty when having difficulties in their program (68.3\%) while PGY 5 residents demonstrated the highest levels (76.1\%) [9].

Is there anything to explain the predictably high rate of resident dissatisfaction in the early years, particularly in the PGY 2 year? One possible explanation can be found in the work of Dr. Walter Menninger with Peace Corps volunteers. In order to facilitate volunteers' readjustment to coming home, he held a series of "completion of service" conferences just prior to the end of their two year assignment. From reviewing nearly 1000 questionnaires and reports of conference discussions, a consistent pattern of psychological adjustment emerged, a pattern subsequently labeled the "morale curve.” See Figure 1. Four "crisis periods" were identified representing major periods of psychological difficulty facing the volunteer. The outcome of the crisis could be positive or negative depending upon the severity of the stress, the individual's coping mechanism, and the support received during the period.

The first crisis period occurs at the beginning of the experience and is characterized as the Crisis of Arrival. Individuals demonstrate high levels of morale, but also apprehension and concern about their ability to meet the challenges. The second critical period is labeled the Crisis of Engagement; at this point the individual is truly engaged in the realities of the situation. Morale com-

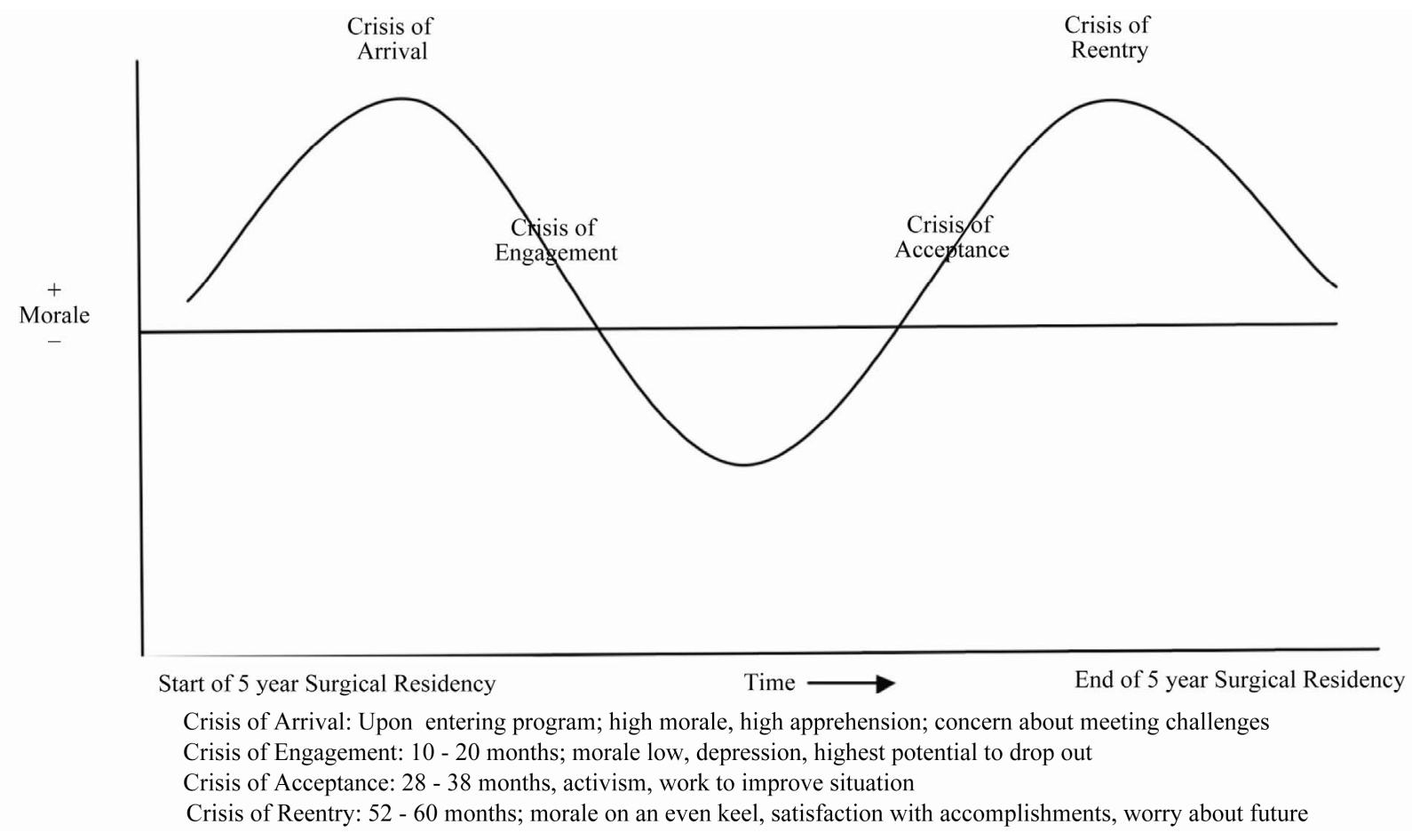

Figure 1. Menninger morale curve. 
monly is low at this point due to frustrations and limited support in the new situation. The central feature of this stage is marked by depression. Individuals take stock of what they left behind, of unrealized expectations of accomplishment, of freedom to express their frustrations. In the Peace Corps study dropouts peaked during this stage. The third phase was noted approximately half-way through the experience and identified as the Crisis of Acceptance. This stage is described as one where morale has been restored as a result of increased knowledge and mastery of the situation. However, emotionally the tone is frequently one of anger and activism. Individuals spoke out more freely and pursued changes to improve the situation. The final crisis is labeled the Crisis of Reentry; morale was generally on an even keel, some satisfaction associated with what they accomplished and with completing their commitment, but some depression associated with giving up their identity and ending relationships, and apprehension about an uncertain future.

Menninger indicated that the morale curve is not unique to the Peace Corps experience, but represents a process that all individuals undergo as they enter new life situations. Morale follows a predictable cycle; how an individual responds is determined by one's position within that cycle. He provides support that shows the model is both universal and scalable (e.g., StaffordClark's description of the changes in the morale of flight crew during their 30-sortie tours in World War II) [10].

To date, there has been no research investigating level of morale of general surgery residents. This study sought to assess level of resident morale in a general surgery residency program at the four crisis points to determine if a cycle similar to the Menninger morale curve is displayed and what the implications might be for resident attrition.

\section{Design, Methodology, and Instrumentation}

The instrument used to measure resident morale was the Morale Assessment in General Practice Index (MAGPI). This is a 14 question self-scoring instrument designed to help physicians think about the sources of stress in their lives and to give them an idea of how they compare with their colleagues. Respondents are asked to select one of three statements that "best reflect how you feel about yourself." The instrument is scored on the basis of three points for the most stressed answer, two for intermediate, and one for least.

MAGPI developers compared their instrument to the General Health Questionnaire 28 (GHQ 28), which is a well-validated measure of distress. Both instruments were sent to 875 physicians in southeast Scotland. Total scores for the MAGPI were well correlated with total GHQ 28 scores $\left(r^{2}=0.68 p<0.001\right)$. In addition, high and intermediate scores in all MAGPI parameters (with one exception) were significantly correlated with GHQ 28 "caseness" - a term used to describe those at risk of psychological stress. Performing a principal component analysis revealed four factors comprising the MAGPI: factor 1-components of control, health, happiness, and support from friends and worries about family; factor 2-components of being up-to-date, perceptions of value of patients and colleagues, and career satisfaction; factor 3-components of getting along with colleagues; factor 4 - concern over alcohol use. When these factors were correlated with GHQ 28, all four factors were correlated highly or moderately, but all demonstrated a $\mathrm{p}$ value of $<0.01$. In summary, the MAGPI seems to stand up particularly well when validated against GHQ 28. Also, the MAGPI was given to 50 General Practitioner trainers. They found it easy to complete and score, felt the questions were relevant, and believed that no important parameters had been missed indicating the MAGPI also has strong face validity and high acceptability [11].

In this study, the MAGPI was administered to a total of 21 residents in their PGY 1, 2, 3, and 5 years. Residents completed the instrument in a timeframe during the 5 year residency cycle congruent with the four crises identified in the Menninger morale curve-PGY 1 residents in August, PGY 2 residents in October, PGY 3 residents in March and PGY 5 residents in May.

Setting

The Hospital of Saint Raphael, General Surgery Residency Program, New Haven, Connecticut. Testing dates were announced approximately one week prior and residents reported to a conference room to complete the instrument.

\section{Participants}

Surgical residents of the Hospital of Saint Raphael General Surgery Residency Program at the PGY 1, 2, 3, and 5 levels.

\section{Results and Statistical Procedures}

Non-parametric methods (Kruskal-Wallis comparisons) were carried out to assess if there were differences in MAGPI responses among the four PGY groups. Even though difference in the MAGPI total means scores did not achieve statistical significance ( $p=0.3229)$, the scores for PGY 1 (16.7) and PGY 5 (17.8) residents were trending lower than PGY 2 (18.0) and PGY 3 (19.5) scores, indicating an overall higher level of morale for the PGY 1 and PGY 5 groups (See Table 1). These findings describe a curve that is similar in shape to the 
Table 1. MAGPI sum scores and factor scores.

\begin{tabular}{ccccc}
\hline MAGPI & PGY 1 & PGY 2 & PGY 3 & PGY 5 \\
\hline MAGPI Sum Scores & 16.7 & 18.0 & 19.5 & 17.8 \\
Factor 1 & 7.1 & 7.7 & 8.3 & 7.3 \\
Factor 2 & 5.3 & 5.0 & 5.2 & 5.1 \\
Factor 3 & 2.3 & 2.7 & 3.3 & 2.9 \\
Factor 4 & 1.0 & 1.0 & 1.0 & 1.0 \\
\hline
\end{tabular}

Menninger morale curve.

Non-parametric analyses of the four factors comprising the MAGPI were also conducted. Factor 1 (components of control, health, happiness, and support from friends and worries about family) yielded mean scores respectively for PGY 1 (7.1); PGY 2 (7.7); PGY 3 (8.3) and PGY 5 (7.3) residents; $(p=0.50)$ (See Table 1). Factor 2 analysis (components of being up-to-date, perceptions of value of patients and colleagues, and career satisfaction) revealed mean scores respectively for PGY 1 (5.3); PGY 2 (5.0); PGY 3 (5.2); and PGY 5 (5.1) residents; $(p=0.91$.). Factor 3 analysis (components of getting along with colleagues) yielded the following mean scores for PGY 1 (2.3); PGY 2 (2.7); PGY 3 (3.3) and PGY 5 (2.9) residents; $(p=0.20)$. Factor 4 (concern over alcohol use) revealed the same mean response (1.0) for all four PGY groups; $(p=1.0)$. All analyses were performed using SAS v.9.1 (SAS Inc.).

\section{Conclusions}

In this surgical residency program, analysis of 21 residents at the PGY 1, 2, 3, and 5 levels who took the MAGPI appear to exhibit different levels of morale based on the amount of time they spent in the residency and in a way that approximates the morale curve described by W. Walter Menninger, M.D. These periods of low morale (PGY 2 and 3 years) also tend to correspond to times when residents are most at risk for leaving a surgery program. Additionally, analysis of the four factors that comprise the MAGPI revealed that factor 1 , which measures components of control, health, happiness, and support from friends and worries about family also described a curve similar to the Menninger Morale Curve with residents displaying the highest levels of morale during the PGY 1 and 5 years and dropping off during the PGY 2 and 3 years. Factor 2 (components of being up-to-date, perceptions of value of patients and colleagues, and career satisfaction) show little variability among the four PGY years tested. This may be due in part to the fact that the question regarding being up to date is poorly correlated with other parameters and the overall score during the pilot of the MAGPI instrument suggested it was not a major cause of stress in doctors' lives [11]. Factor 3 analysis (components of getting along with colleagues) indicated that PGY 1 residents demonstrated higher levels of morale than PGY 2 and PGY 3 residents while PGY 5 residents demonstrated higher levels of morale than just PGY 3 residents. Factor 4 analysis (concern over alcohol use) were answered "I have no problems with alcohol" by 20 of 21 residents in the study. As a result, there were no differences in morale between the groups. It may be residents were concerned about test anonymity and confidentiality and did not want the residency program uncovering a potential issue. The study by McKinstry, et al., indicates that although the MAGPI is generally a reliable instrument, the authors questioned the usefulness of the item related to alcohol use. This question demonstrated the lowest correlation (0.21) of any of the four factors with the GHQ and the worst response for this item ("I am worried about my use of alcohol") was not significantly associated with "caseness" [11].

\section{Discussion}

To date, there are no studies in graduate medical education residency programs that have sought to compare resident level of morale with that described by the Menninger morale curve. Based on the above findings, lower levels of morale seen in PGY 2 and 3 level residents and higher levels of morale demonstrated by PGY 1 and PGY 5 residents are congruent with the four crisis periods described by Menninger in his morale curve [10]. This tends to reinforce Menninger's claim that the pattern of morale represents a process applicable to all individuals as they enter a new life situation like surgical residency.

These periods of low morale (PGY 2, PGY 3) coincide with times when residents are at higher risk for leaving surgical residency. For that reason, programs should pay particular attention to residents at these stages in their residency for cues related to severity of stress, coping mechanisms, and support they receive from colleagues, faculty and staff.

Studies have demonstrated that people who handle stress effectively have a greater sense of control over what occurs in their lives and remain healthier than those who feel powerless in the face of external events. Using cognitive control to incorporate stressful events into a life plan rather than taking a narrow, focused approach, mitigates the jarring effects of those events. For residents at risk who do not demonstrate a high level of "cognitive control", a strategy of "anticipatory guidance" can be used to help these residents. This is using the healthy defense mechanism of anticipation to improve the resident's capacity to adapt to a new life situation or situational crisis. Making future likelihoods known to these residents helps them cope with new situations and miti- 
gates the fear and anxiety of upcoming events that are expected to be stressful. It helps individuals put the situation in proper perspective and reduce the sense of isolation and helplessness that they may experience. Residents who have gone through a similar experience are helpful. Successful support programs should foster opportunities for individuals to be in control of their lives, to be committed to some activity, and to address the challenge in ways that permit sublimation of the conflicted feelings generated by the life change-through work, play, and personal relationships [10].

\section{Recommendations for Further Study}

After having completed the research and having correlated findings, conclusions, and implications, the following recommendations are made:

1) Continued research should be directed toward determining linkages between resident morale and their positions in the residency program experience.

2) Attempts should be made to duplicate this study with larger sample sizes and in programs of different duration.

3) Longitudinal research should be conducted tracking the same residents as they progress through a residency program.

\section{References}

[1] R. Bell, M. Banker, R. Rhodes, T. Biester and F. Lewis, "Graduate Medical Education in Surgery in the United States," Surgical Clinics of North America, Vol. 87, No. 4, 2007, pp. 811-823. doi:10.1016/j.suc.2007.06.005

[2] D. A. Andriole, et al., "Attrition during Graduate Medical Education,” Archives of Surgery, Vol. 143, No. 12, 2008, pp. 1172-1177. doi:10.1001/archsurg.143.12.1172

[3] T. F. Dodson and A. L. Webb, "Why do Residents Leave General Surgery? The Hidden Problem in Today’s Pro- grams,” Current Surgery, Vol. 62, No. 1, 2005, pp. 128-131. doi:10.1016/j.cursur.2004.07.009

[4] R. P. McAlister, D. A. Andriole, S. E. Brotherton and D. B. Jeffe, “Attrition in Residents Entering U.S. Obstetrics and Gynecology Residencies: Analysis of GME Census Data," American Journal of Obstetrics and Gynecology, Vol. 199, No. 5, 2008, pp. 574e1-574e6.

[5] W. Longo, J. Seashore, A. Duffy and R. Udelsman, “Attrition of Categoric General Surgery Residents: Results of a 20-Year Audit,” The American Journal of Surgery, Vol. 197, No. 6, 2009, pp. 774-778. doi:10.1016/j.amjsurg.2008.06.038

[6] D. C. Lynge, E. H. Larson, M. J. Thompson, R. A. Rosenblatt and L. G. Hart, “A Longitudinal Analysis of the General Surgery Workforce in the United States 1981-2005," Archives of Surgery, Vol. 143, No. 4, 2008, pp. 345-350. doi:10.1001/archsurg.143.4.345

[7] C. B. Everett, S. D. Helmer, J. S. Osland and R. S. Smith, "General Surgery Resident Attrition and the 80-Hour Workweek,” The American Journal of Surgery, Vol. 194, No. 6, 2007, pp. 751-757. doi:10.1016/j.amjsurg.2007.08.033

[8] T. J. Leibrandt, C. M. Pezzi, S. A. Fassler, E. F. Reilly and J. B. Morris, "Has the 80-Hour Work Week Had an Impact on Voluntary Attrition in General Surgery Residency Programs?" Journal of The American College of Surgeons, Vol. 202, No. 2, 2006, pp. 340-344. doi:10.1016/j.jamcollsurg.2005.09.018

[9] H. Yeo, et al., “Attitudes, Training Experiences, and Professional Expectations of US General Surgery Residents," Journal of the American Medical Association, Vol. 302, No. 12, 2009, pp. 1301-1308. doi:10.1001/jama.2009.1386

[10] W. W. Menninger, “Adaptation and Morale, Predictable Responses to Life Changes," Bulletin of the Menninger Clinic, Vol. 52, No. 3, 1988, pp. 198-210.

[11] B. McKinstry, M. Porter, R. Wrate, R. Elton and J. Shaw, "The MAGPI (Morale Assessment in General Practice Index): A New Way for Doctors to Self-Assess Their Morale,” Education for Primary Care, Vol. 15, No. 2, 2004, pp. 231-241. 